\title{
Modelos animales de miedo y ansiedad: descripciones neuro-conductuales
}

\author{
Animal models of fear and anxiety: \\ neurobehavioral descriptions
}

\author{
Andrea Mora-Gallegos ${ }^{1}$ \\ Sofía Salas Castillo ${ }^{2}$ \\ Universidad de Costa Rica, Costa Rica
}

\begin{abstract}
Resumen. Los modelos animales de miedo y ansiedad se han utilizado para la comprensión de trastornos de ansiedad en humanos, sin embargo no ha sido fácil diferenciar ambos conceptos a nivel fisiológico y conductual. Una forma de modelar estos trastornos es mediante pruebas conductuales de ansiedad (como el laberinto en cruz elevado o la prueba de campo abierto) y de miedo (como el condicionamiento al miedo y la evitación activa). Además, estos modelos permiten estudiar diferentes áreas cerebrales involucradas como la amígdala, el hipocampo y la corteza prefrontal. En general, el miedo y la ansiedad se pueden considerar respuestas adaptativas normales, sin embargo niveles elevados de ambos pueden generar consecuencias perjudiciales para el individuo.
\end{abstract}

Palabras clave. Modelos animales, ansiedad, miedo, comportamiento.

Abstract. Animal models of fear and anxiety have been widely used for the comprehension of anxiety disorders in humans, however, it has not been easy to distinguish between both concepts at physiological and behavioral levels. One way to model anxiety disorders is through behavioral tests of anxiety, (such as the elevated plus maze and the open field test), and fear (using the fear conditioning paradigm and active avoidance). Furthermore, animal models are relevant to study the involvement of different brain areas, like the amygdala, hippocampus and prefrontal cortex. In general, fear and anxiety can be considered as normal adaptive responses; however, elevated levels of both might generate detrimental consequences for the individual.

Keywords. Animal models, anxiety, fear, behavior.

\footnotetext{
${ }^{1}$ Andrea Mora-Gallegos. Centro de Investigación en Neurociencias, Universidad de Costa Rica. Direccion postal: Univeridad de Costa Rica, Vicerrectoría de Investigación, 11501-2060, San José, Costa Rica. E-mail: andreamorag@gmail.com

${ }^{2}$ Sofía Salas. Centro de Investigación en Neurociencias, Universidad de Costa Rica. E-mail: sosc459@gmail.com
} 


\section{Introducción}

La definición de los términos "miedo" y "ansiedad" puede resultar compleja debido a su traslape a nivel fisiológico, conductual y cerebral. En este artículo se buscará delimitar estos términos, así como su forma de modelarlos, reproducirlos e interpretarlos en animales, dando énfasis a nivel neuro-conductual. A pesar de las dificultades para diferenciar los conceptos, se considera que es posible modelarlos diferencialmente, lo cual se intentará en el desarrollo de la temática.

El término ansiedad proviene del latín anxietas refiriéndose a un estado de agitación, inquietud o zozobra, y se relaciona con la previsión de peligros futuros e indefinibles. La ansiedad es una respuesta difusa, caracterizada por la intranquilidad e hipervigilancia, que puede desembocar en aprehensión y activación del sistema nervioso autónomo en un individuo en ausencia de un estímulo específico que la desencadene (Gómez, Saldívar \& Rodríguez, 2002; Sierra, Ortega \& Zubeidat, 2003). En animales, específicamente en ratas y en ratones, reacciones como la hipervigilancia, la evitación y el escape, se consideran respuestas adaptativas normales, pero podrían convertirse en perjudiciales si se exacerban (Gómez et al., 2002).

Por otro lado, el miedo ha sido considerado un mecanismo defensivo de un individuo ante un estímulo peligroso. Las respuestas ante una situación que genera miedo son esenciales en la protección del sujeto frente a ese peligro (Kim \& Whan, 2006). Por consiguiente, aprender a reconocer la relación entre un estímulo aversivo y el ambiente en que éste se presenta (condicionamiento al miedo) permite desarrollar patrones de comportamiento apropiados en respuesta a diferentes amenazas y así convertirse en un factor esencial para la sobrevivencia (Blanchard, Blanchard, Griebel \& Nutt, 2008; Bruchey, Jones \& Monfils, 2010; Maren, 2001; Sullivan \& LeDoux, 2004). El miedo puede considerarse una de las experiencias emocionales más potentes en la vida.

El poder que tiene esta experiencia es crítica en cuanto a la sobrevivencia de los vertebrados, puesto que activa todo el sistema defensivo de comportamiento, que sirve a su vez como protección contra amenazas en el ambiente (Fendt \& Fanselow, 1999).

Walter Canon contribuyó de manera importante al desarrollo del estudio del miedo y la ansiedad con su trabajo sobre la relación entre respuestas emocionales y la reactividad fisiológica (Gómez et al., 2002). Canon describió la respuesta de "pelear-huir" ("fight-flight"), que algunos otros investigadores la denominan como la respuesta de "congelamientopelea-huida" ("freeze-fight-flight"), pues en el miedo, la conducta de congelamiento ("freezing") siempre aparece, aunque sea de manera breve, en presencia de un estímulo que se interpreta como peligroso (Sullivan \& LeDoux, 2004). Como se mencionó anteriormente, esta conducta se considera ventajosa a nivel evolutivo debido a que ayuda a evitar a depredadores, optimiza procesos atencionales y de percepción y prepara al organismo para un rápido escape o una posible pelea (Hagenaars, 2014). De hecho, la investigación reciente en seres humanos ha reconocido la importancia de la conducta de congelamiento o "freezing" como parte de la cascada defensiva humana (Hagenaars, 2014).

De esta manera, tanto el miedo como la ansiedad preparan al organismo para hacer frente a situaciones de peligro, con la diferencia de que en el miedo, existe un peligro que es reconocible por el individuo (existe un estímulo definido, presente, que lo desencadena) (Blanchard et al. 2008; Gómez et al., 2002; Zeidner \& Matthews, 2011). La ansiedad corresponde a un estado de incertidumbre y está orientada casi siempre hacia una previsión futura de posibles problemas, peligros, entre otros; que en muchos casos, están fuera del control del individuo y que generan tanto síntomas mentales como físicos (Zeidner \& Matthews, 2011). De hecho, en muchas ocasiones, la ansiedad antecede al miedo, y en otras la ansiedad es más frecuente después de experimentar miedo (Zeidner \& Matthews, 2011).

Es importante mencionar que en seres humanos, el estudio del miedo y la ansiedad, ha resultado difícil de esclarecer a niveles que tengan una importancia y relevancia etológica, debido por ejemplo a que es difícil encontrar pacientes con daño bilateral en la amígdala, región que se ha considerado esencial tanto para animales como para 
humanos (Bechara, 1995; La Bar, 1995), aspecto que se desarrollará más adelante y que se ha intentado subsanar con la implementación de modelos animales.

\section{Modelo animal de miedo y ansiedad}

Los modelos animales han sido una herramienta clave en la investigación, pues han permitido esclarecer procesos fisiológicos, bioquímicos, conductuales y demás, que se asemejan a los procesos respectivos en el ser humano. Lo que ha permitido alcanzar una mayor comprensión en gran variedad de campos, así como predicciones más acertadas en cuanto a respuestas del organismo ante diversos estímulos, ambientes y tratamientos (Hau, 2003).

La problemática a nivel de predicciones de los modelos animales se basa, en su mayor parte, en arriesgadas interpretaciones de los resultados obtenidos. Es fundamental, para no cometer tales errores, conocer las limitaciones de cada modelo, tales como las diferencias entre especies, la intervención de otros factores no asociados con la condición bajo estudio, la falta de un modelo que nos replique la totalidad de las características presentadas en el ser humano (Hau, 2003; Shanks, Greek \& Greek, 2009), además de las dificultades de replicar funciones humanas complejas (Shanks et al., 2009). Ante este panorama, se ha buscado asociar conductas propias de la biología animal, que respondan de una forma similar a la conducta análoga en el ser humano. Tal ha sido el caso para estudios sobre miedo y ansiedad (Rohra \& Qazi, 2008).

Una de las pruebas que ha demostrado ser sumamente útil para relacionar miedo y ansiedad, es la prueba de condicionamiento al miedo, la cual sigue los principios básicos del condicionamiento clásico propuesto por Pavlov; el condicionamiento clásico (Maren, 2001) o condicionamiento Pavloviano se basa en el proceso por el cual un estímulo neutro o también llamado estímulo inocuo o estímulo condicionado (EC), puede adquirir propiedades emocionales (en el caso de condicionamiento al miedo) si ocurre simultáneamente con un estímulo significativo biológicamente, denominado estímulo incondicionado (EI) (Sullivan \& LeDoux, 2004).
En animales, esta prueba se trata de presentar un estímulo neutro, tal como una luz o un sonido, seguido inmediatamente por un estímulo adverso como lo sería un shock eléctrico leve en las patas (por lo general $0.5 \mathrm{~mA}$ ), el cual induce una respuesta incondicionada de miedo. Bajo estas condiciones el estímulo neutro será un estímulo condicionado al ser asociado con uno adverso o incondicionado. De esta manera, después de un corto entrenamiento se obtendrá también una respuesta de miedo aún en presencia de solo el estímulo condicionado por una anticipación del estímulo incondicionado (Akirav \& Maroun, 2007; Fendt \& Fanselow, 1999).

Por su parte, en seres humanos existen teorías que han implicado al condicionamiento al miedo en la patogénesis de desórdenes de ansiedad desde hace al menos 80 años, donde la investigación básica con animales ha generado aportes esenciales relacionados con el condicionamiento al miedo en humanos (Lissek, 2005). De hecho, existe evidencia que apoya que existen áreas cerebrales similares en el aprendizaje del miedo en humanos (Lissek, 2005). En este sentido, la clarificación de diferencias en el condicionamiento al miedo aplicado a pacientes con trastornos de ansiedad y controles saludables, es posible para el beneficio de futuros esfuerzos que ayuden a dilucidar aspectos neurobiológicos de la ansiedad clínica (patológica) (Lissek, 2005).

Algunos autores han intentado desarrollar teorías en relación con el condicionamiento al miedo y la ansiedad clínica o patológica. Por ejemplo Eysenck (1979, citado por Lissek, 2005), propone que la ansiedad patológica es resultado del fallo en extinguir la respuesta de miedo condicionado. De hecho, él formula que pacientes ansiosos, adquirirán una mayor respuesta de miedo comparados con controles, que resultarán en un mayor reforzamiento del estímulo condicionado (una reacción mayor) en ausencia del incondicionado. En consecuencia, estas personas tendrán un peor desempeño y una mayor resistencia en pruebas de extinción que sus respectivos controles (Lissek, 2005). Otra propuesta en este sentido, fue desarrollada por Davis (2000), en la que se propone que la ansiedad patológica puede ser también el resultado del fallo en 
la inhibición de la respuesta de miedo en presencia de señales que lo eviten. Además, Seligman (1971) propone una teoría de la "preparación", que afirma que los humanos están biológicamente preparados para formar asociaciones aversivas hacia estímulos que han sido relevantes a través de la evolución, como serpientes, arañas, espacios cerrados, entre otros, (Lissek, 2005).

Según esta teoría, el ser humano está predispuesto a adquirir fobias y miedos que significaron amenazas para antecesores como predadores, o espacios ampliamente abiertos que a objetos y situaciones que se han originado de forma más contemporánea (Mineka \& Ohman, 2002). Estos datos han sido aplicados en diversos laboratorios donde se ha implementado el condicionamiento al miedo comparando imágenes de estímulos como arañas o serpientes con imágenes como flores o figuras geométricas (EC) junto con shocks eléctricos.

El registro fisiológico más frecuentemente utilizado en estudios con seres humanos es la respuesta de conductancia de la piel, aunque también se han utilizado medidas como el pulso, la frecuencia cardiaca, respuestas corticales (ondas cerebrales) y medidas cognitivas subjetivas, como por ejemplo calificación de rangos de aversividad de estímulos (Mineka \& Ohman, 2002), sin embargo, este tema ha sido poco explorado.

Esta evidencia demuestra lo relevante del uso de modelos animales, dada su utilidad en seres humanos resumida de la siguiente manera. En primer lugar, se utilizan los modelos donde se induce el miedo con el fin de entender cómo influye en el comportamiento. En segundo lugar, la investigación de las bases neuroanatómicas y neuroquímicas del miedo y la ansiedad son un requisito para desarrollar estrategias para el tratamiento y la cura de trastornos de ansiedad. En tercer lugar, experiencias relacionadas con el miedo son rápidamente aprendidas y recordadas por largos periodos de tiempo. Por lo tanto, estos modelos se han convertido también en un excelente medio para el estudio de los procesos y mecanismos subyacentes al aprendizaje y la memoria emocional (Fendt \& Fanselow, 1999).

\section{Evaluación conductual del miedo y la ansiedad en modelos animales}

Existen diversos modelos animales en el estudio del miedo y la ansiedad, sin embargo la mayoría se enfoca en el estudio del comportamiento debido a la importancia evolutiva que se le ha dado, aunque se han realizado estudios también relacionados con aspectos fisiológicos y farmacológicos (Campos, Fogaça, Aguiar \& Guimaraes, 2013). En este sentido, la ansiedad ha sido relacionada principalmente con la evaluación de riesgo, evocado en circunstancias donde hay un peligro potencial o ambiguo. De hecho, el diazepam (medicamento ansiolítico), parece afectar la evaluación del riesgo, pero no la conducta de "freezing", relacionada principalmente con el miedo, en situaciones que no tienen escapatoria (Hagenaars, 2014).

A continuación, se mencionarán algunas pruebas conductuales utilizadas en ratas y ratones para medir la ansiedad, como lo son el laberinto en cruz elevado, la prueba de campo abierto; y para evaluar el miedo, como la prueba de condicionamiento al miedo y de evitación activa.

Laberinto en Cruz Elevado. El laberinto en cruz elevado (LCE) ha sido utilizado para medir el comportamiento incondicionado o espontáneo en roedores, creando un ambiente de aproximación y evitación (Rodgers \& Dalvi, 1997). El LCE contiene dos brazos abiertos y dos brazos cerrados y se evalúa el tiempo y la frecuencia de entradas a ellos. Además, se mide el "head-deeping" (que consiste en asomarse por debajo de los brazos abiertos), la postura de estiramiento, la exploración, la locomoción y el acicalamiento como indicadores del nivel de ansiedady de la evaluación sobre la exploración en un ambiente desconocido (Walf \& Frye, 2007). Como se mencionó, en esta prueba el animal tiene el conflicto entre la evitación y el acercamiento, ya que el animal evita espacios abiertos donde, de acuerdo con la etología del roedor, hay mayor posibilidad de depredación, sin embargo, su conducta exploratoria lo motiva a recorrer los brazos abiertos (Becerra-García, Madalena, Estanislau, Rodríguez-Rico \& Dias, 2007; Bessa et al., 2005; Litvin et al., 2008). 
El laberinto en cruz elevado ha sido ampliamente utilizado por sus ventajas en cuanto a la facilidad de la aplicación de la prueba, ya que en general se trata de una prueba simple que no requiere entrenamiento del animal (McNaughton \& Zangrossi, 2008). Además, se ha validado el uso de esta prueba mediante el efecto de algunos fármacos ansiolíticos como lo es el caso del diazepam en particular, y las benzodiacepinas en general.

Se ha visto que este tipo de fármacos incrementan las entradas a brazos abiertos, mientras que fármacos ansiogénicos, como agonistas de receptores de 5-HT1B/2C, antagonistas de receptores de GABAA, ocasionan el efecto contrario (Hogg, 1996; Rodgers \& Dalvi, 1997, Carobrez \& Bertoglio, 2005). Asimismo, esta prueba se ha utilizado para medir emocionalidad relacionada con algunos desórdenes de ansiedad como la ansiedad generalizada, fobias o estrés postraumático (Carobrez \& Bertoglio, 2005).

En general, se pueden resumir las características positivas del LCE de la siguiente manera: es una prueba sencilla y rápida de aplicar (se realiza una sola prueba de 5 minutos) y no se requiere equipo costoso; se basa en el comportamiento espontáneo, por lo que no hay entrenamiento; no requiere de privación de agua y alimento para el animal, y es útil para identificar los efectos de los ansiolíticos o benzodiacepinas (Pellow, 1985; Rodgers et al, 1997; Carobrez \& Bertoglio, 2005).

Prueba de Campo Abierto. La prueba de campo abierto también ha sido una prueba ampliamente utilizada para medir ansiedad. Es un recinto generalmente cuadrado, rectangular o circular con paredes alrededor que impiden la salida o escape del animal (Gould, Dao \& Kovacsics, 2009). Desde los estudios originales de Hall en 1934, la prueba de campo abierto ha sido utilizada para medir las reacciones de los roedores en un ambiente que no tiene salida (Suárez, 1981). En los diferentes laboratorios la prueba varía en cuanto a su duración que puede ir desde dos minutos hasta 30 minutos. En este periodo de tiempo, lo que se analiza es el comportamiento exploratorio y la respuesta ante ese ambiente "novedoso" (Archer, 1973; Gould et al, 2009).
Hall además fue el primer investigador que utilizó el término "emocionalidad" en 1934 para conceptualizar cambios conductuales debido a una alta actividad del sistema nervioso simpático (Archer, 1973). Por consiguiente, los primeros estudios se basaron en la medición de la defecación como un criterio de emocionalidad y activación de ese sistema; sin embargo, poco a poco se han ido agregando otras variables como la locomoción, el "rearing" o exploración vertical (Suárez, 1981) y el acicalamiento en la prueba de campo abierto (Roth, 1979). De hecho, en estudios realizados en nuestro laboratorio, la medición del acicalamiento, la distancia y velocidad del desplazamiento han demostrado ser de utilidad para estudiar los niveles de ansiedad en este paradigma conductual (Brenes, Rodríguez \& Fornaguera, 2006; Masís-Calvo, SequeiraCordero, Mora-Gallegos \& Fornaguera-Trías, 2013; Sequeira-Cordero, Masís-Calvo, Mora-Gallegos \& Fornaguera-Trías, 2013).

Otro indicador que se ha relacionado con la ansiedad en esta prueba es el grado de habituación a la misma. La habituación representa un aprendizaje fundamental en el cual el organismo disminuye la respuesta ante un estímulo redundante no significativo (Dubovicky, 2004; Walsh \& Cummins, 1976). En una prueba como el campo abierto, la habituación se manifiesta como un decremento gradual en la actividad locomotora (Dubovicky, 2004; Walsh \& Cummins, 1976). Además, según Pleskacheva (1996) la exposición prolongada al campo abierto induce habituación hacia la novedad y existe una tendencia a que conforme pase el tiempo en un campo abierto el acicalamiento va aumentando, debido a una habituación en respuesta a una situación.

Las investigaciones con esta prueba se basan en la medición de la frecuencia y la cantidad de tiempo que el animal pasa en el área central y en el área periférica del campo (Prut \& Belzung, 2003). Se asume que los roedores siempre van a preferir el área periférica, que por lo general es más oscura y donde el animal se encuentra menos expuesto, sin embargo, al igual que en el laberinto en cruz elevado, su conducta exploratoria innata lo lleva también a explorar el área central (Litvin et al., 2008). De acuerdo con la exploración 
y locomoción del animal, así como entradas al área central, se ha determinado el nivel de ansiedad, donde se espera que los sujetos menos ansiosos pasen más tiempo en el área central y disminuyan la exploración y la locomoción a lo largo de la prueba (Becerra-García et al., 2007; Ossenkopp \& Mazmaninan, 1985).

De hecho, autores como Prut et al. (2003), afirman que si el tamaño del campo abierto es suficientemente grande en relación con los animales, se pueden medir indicadores de agorafobia (temor a los espacios abiertos). También se le ha dado otras aplicaciones, como por ejemplo para evaluar efectos sedantes, tóxicos o estimulantes de ciertos compuestos. Son numerosos los usos de esta prueba lo que ha permitido caracterizar el comportamiento de los roedores en un ambiente inocuo (Gould et al, 2009).

Condicionamiento al miedo. En la prueba de condicionamiento al miedo, descrito anteriormente, se presentan algunas respuestas conductuales; una de ellas es la respuesta de sobresalto que se caracteriza por movimientos abruptos y rápidos y se mantiene por unos segundos más después de que se termina el estímulo incondicionado (descarga eléctrica en las patas). Generalmente, la respuesta seguida por esta conducta es la de "freezing", en donde el animal está inmóvil, visiblemente alterado dada su respiración agitada, piloerección y tensión muscular evidente (Fanselow \& Ponnusamy, 2008).

El estímulo incondicionado y la respuesta consecuente son un claro ejemplo de miedo, donde el efecto adverso se presenta y el animal muestra la conducta de sobresalto al recibir el estímulo. Lo anterior conlleva a una evaluación de riesgo por parte del animal, en donde se maneja la probabilidad de que el evento se repita, la ansiedad sería consecuencia de la probabilidad de recurrencia, con una relación proporcional a esta (Becerra-García et al., 2007).

De hecho, algunos autores afirman que con una sola exposición al estímulo condicionado en presencia del incondicionado, se puede observar el aprendizaje con algunas respuestas defensivas específicas de la especie, pues el miedo es una de las conductas que se aprende más rápidamente, la cual es más recordada a lo largo del tiempo debido a su contenido emocional, puede mantenerse incluso durante toda la vida (Burman, Hamilton \& Gewirtz, 2010; Fanselow \& Ponnusamy, 2008; Fendt \& Fanselow, 1999; Gale, Anagnostaras, Godsil, Mitchell, Nozawa, Sage, Wiltgen \& Fanselow, 2004; Roozendal, McEwen \& Chattarji, 2009).

Prueba de evitación activa. El aprendizaje de respuestas instrumentales (operantes) es otra forma de enfrentar situaciones peligrosas, de modo que es una conducta evolutivamente beneficiosa que se observa en muchas especies (Wendler et al., 2014). Para evaluar esta respuesta se ha utilizado una prueba conductual en la que el animal tiene la posibilidad de impedir el estímulo aversivo, es decir, aprende poco a poco a evitar la descarga eléctrica en sus patas. A esta prueba se le conoce como evitación "activa", porque el animal debe ejecutar una conducta para poder evitar el estímulo incondicionado (Choi, Cain \& LeDoux, 2010). Esta conducta se espera que sea emitida en presencia del estímulo condicionado, casi siempre, un tono (Moscarello \& LeDoux, 2013), pues este estímulo le genera "pistas" al animal para anticipar un peligro.

Además, ambos tipos de pruebas (el condicionamiento propiamente y la evitación activa), provocan dos tipos de conducta contrarias, ya que cuando el animal se condiciona al miedo, emite la conducta de "freezing", mientras que en la evitación activa el animal debe movilizarse para evitar el EI (Moscarello \& LeDoux, 2013). Por supuesto, para que un animal evite un estímulo aversivo teniendo "pistas" de su anticipación (por ejemplo, el sonido), debe primero aprender las pistas específicas que predicen ese estímulo. En este caso, la prueba de evitación activa se utiliza para que el animal evada la descarga eléctrica cruzando al lado opuesto de la caja (Boschen et al., 2011; Choi et al., 2010; Pang et al., 2011; Wendler, 2014), mientras que en el condicionamiento al miedo explicado anteriormente, el animal no puede evitar el EI.

Para esta prueba los protocolos utilizados constan de una primera fase de entrenamiento en la que se presenta un estímulo neutro, inmediatamente seguido de un estímulo aversivo, como la descarga eléctrica en 
las patas, con una duración de entre 0.5 y 20 segundos, según el objetivo del estudio en cuestión (Boschen et al., 2011; Moscarello \& LeDoux, 2013). En esta fase de entrenamiento se busca que el animal haga la asociación entre ambos estímulos, de modo que, durante la fase de prueba, éste efectúe la misma conducta de evitación en presencia del estímulo neutro o condicionado (Moscarello \& LeDoux, 2013; Choi et al., 2010).

Las estrategias de evitación que un animal desarrolla son importantes porque el sujeto disminuye el miedo y la ansiedad relacionados con esta prueba e incluso en otros contextos donde se presente el EC (Choi et al., 2010; Moscarello \& LeDoux, 2013). La evitación activa, apropiada a la situación, también se considera, además del "freezing", una estrategia exitosa para lidiar con el peligro en ratas. De hecho, en humanos, la evitación es utilizada por pacientes con desórdenes de miedo, para reducir su exposición a situaciones que provoquen miedo o ansiedad (Choi et al., 2010; Wendler, 2014).

Todas estas pruebas, no solamente han sido útiles para estudiar el comportamiento, sino que han permitido dilucidar regiones cerebrales involucradas (Carobrez \& Bertoglio, 2005), de las cuales se profundizará a continuación.

\section{Regiones cerebrales involucradas en el miedo y la ansiedad}

El substrato neural del aprendizaje y la memoria emocional en animales, ha sido ampliamente estudiado; en este sentido, la amígdala (AMG) es un componente esencial en una red neural de reacciones emocionales (Ganella \& Kim, 2014). Sin embargo, este modelo de formación de la memoria y su relación con los mecanismos cerebrales ha sido poco estudiado en seres humanos, por lo que lo relativo a las bases neurales del procesamiento de la información emocional se ha obtenido principalmente de animales (La Bar, 1995).

La AMG está conformada por diferentes núcleos con funciones específicas para la formación de la memoria emocional. La amígdala lateral (AL) reúne la información sensorial y es donde ocurre la asociación del estímulo condicionado con el incondicionado. Es decir, la gran plasticidad de este núcleo permite la detección de un estímulo neutro como un estímulo aversivo, induciendo la respuesta de miedo. La AL, a su vez, se comunica con el núcleo central de la amígdala (NCeA), punto que cuenta con la mayor cantidad de eferencias que se encargan de generar la respuesta al miedo, tanto conductual como autonómica y endocrina (Rodrigues, LeDoux, \& Sapolsky, 2009).

Además de la AMG otras regiones del sistema límbico como el hipocampo (HPC) y la corteza prefrontal (CPF) han demostrado jugar un rol significativo en situaciones de miedo y ansiedad (Akirav \& Maroun, 2007; Engel, Bandelow, Gruber, \& Wedekind, 2009; Moustafa et al., 2013). El HPC parece estar directamente involucrado en la generación de una respuesta ante la situación adversa, principalmente en el conflicto de pelea-huida ("fight flight"). El HPC es responsable de aumentar el estado de vigilancia y atención en el sujeto, así como en procesar la información del contexto (Canteras \& Graeff., 2014). Además, el HPC también puede modular la actividad de la AMG y la CPF, favoreciendo la integración de la respuesta al miedo. A su vez, el estado emocional y motivacional y su procesamiento en la AMG, va a alterar de forma importante el funcionamiento del HPC (Canteras \& Graeff., 2014; Radulovi \& Tronson, 2010).

Como se mencionó previamente, las funciones cognitivas pueden verse moduladas por el miedo y la ansiedad; en este sentido, la participación de la $\mathrm{CPF}$ es esencial en el restablecimiento del equilibrio después de un evento estresante (Arnsten, 2009). Si bien esta estructura no tiene un rol en la adquisición de la memoria emocional, sí es importante para la respuesta emocional aprendida, para la cual ocurre una constante retroalimentación entre esta y la AMG (Marek, Strobel, Bredy \& Saj, 2013).

De hecho, existe el llamado conflicto emocional, que se basa en la lucha entre una repuesta rápida ante un estímulo impredecible a cargo de la AMG, y una respuesta mediada por un alto componente cognitivo favorecida por la CPF. En la resolución de este conflicto, la corteza cingulada anterior (ACC) va a reducir la actividad de la amígdala, permitiendo mayor actividad de la CPF (Engel et al., 2009). Como parte de la respuesta a una amenaza, la AMG también favorece el aumento de las concentraciones de glucocorticoides, 
que a su vez estimulan la actividad de la amígdala e inhiben el funcionamiento de la CPF. Esta modulación de la actividad favorece un control de la respuesta al estrés por parte de la amígdala, comprometiendo parte del control cognitivo a causa de la disminución en la actividad de la CPF (Arnsten, 2009; Green \& McCormick, 2013; Rodrigues et al., 2009).

Cuando un estímulo o una situación son interpretados como amenazadores, el cerebro activa algunos circuitos neuronales para adaptarse a las demandas. Dos de esos circuitos se relacionan con el sistema nervioso autónomo y la liberación de glucocorticoides (por medio de la activación del eje HPA). Por ejemplo, cuando aparece la conducta de "freezing" en el sistema nervioso autónomo se activa tanto la rama simpática como la parasimpática en mayor o menor grado dependiendo del momento (Hagenaars, 2014).

La activación de la rama simpática se ve reflejada en un incremento de la "vigilancia" y de síntomas físicos como: incremento en la presión arterial, incremento del tono muscular, hiper-responsividad y un aumento en la conducta de sobresalto. Además, este sistema utiliza la adrenalina y noradrenalina, que actúan en el cerebro en lugares que controlan la atención y acciones de respuesta como respuestas de "pelea-huida" y comportamiento de "freezing". Por otro lado, la rama parasimpática causa una desaceleración del ritmo cardiaco (Hagenaars, 2014).

El otro nivel de activación se relaciona con la liberación de glucocorticoides; los cuales han sido identificados como los compuestos a través de los cuales se integran, la ansiedad y el miedo (Grillon et al., 2007). Ante un estímulo adverso de observa un incremento en la actividad cerebral, así como en el sistema nervioso periférico por liberación de glucocorticoides (Rodrígues et al., 2009).

Grillon et al. (2007) relacionaron los glucocorticoides con la sensibilización a la ansiedad, lo cual ocurre de manera paralela a una potenciación del miedo, durante el cual se da una activación del eje Hipotalámico-PituitarioAdrenal (HPA). La activación de este eje comienza en el núcleo paraventricular del hipotálamo (NPV), el cual aumenta la liberación de CRF, también llamado $\mathrm{CRH}$ (del inglés, Corticotropine Releasing Factor o Corticotropine Releasing Hormone) en la circulación del sistema denominado "porta-hipofisiario". El CRF entonces estimulará a la pituitaria anterior aumentando así la secreción de ACTH (hormona adrenocorticotropa) hacia la circulación general.

La ACTH actúa sobre la corteza adrenal para inducir la síntesis de glucocorticoides e incrementar su secreción (específicamente corticosterona en roedores). En condiciones normales, esta elevación de los niveles de glucocorticoides activará receptores de glucocorticoides, que servirán, entre otras, para disminuir y hasta eliminar la secreción de CRF y ACTH tanto en hipotálamo como en la hipófisis (proceso conocido como retroalimentación negativa) y así llevar al organismo nuevamente a sus niveles basales (Duval, González, \& Rabia, 2010; Grillon et al., 2007; Hagenaars, 2014; Levine, 2005). Los efectos de los glucocorticoides no se relacionan únicamente con la regulación del eje HPA, sino que además intervienen en funciones del cuerpo, como la respuesta inmune. Se ha visto que la secreción prolongada de los glucocorticoides puede provocar, entre otros, inmunosupresión, disfunción autonómica y atrofia de algunos tejidos (Fulford \& Harbuz, 2005).

\section{Conclusiones}

Entender lo que sucede a nivel sináptico, fisiológico y conductual por medio de modelos animales de miedo y de ansiedad, ha permitido comprender procesos similares en seres humanos (Sullivan \& LeDoux, 2004); lo que a su vez favorece el diseño de nuevas y más efectivas formas de terapia para el manejo de trastornos asociados (Castro \& Landeira, 2008). Los modelos animales de ansiedad y miedo, aún cuando no logran una replicación completa de las características presentadas en el ser humano, han demostrado tener una alta validez en relación con patrones de respuesta y áreas cerebrales involucradas, lo que los hace herramientas de estudio muy atinadas. Además, la descripción etológica del miedo y ansiedad en animales, concuerda con aquella observada en humanos. Igualmente, a nivel fisiológico, 
la activación de la AMG y sus repercusiones a otros sistemas, como el sistema nervioso autónomo y el eje HPA, son sumamente similares (Hagenaars, 2014).

Estas grandes concordancias entre la respuesta observada en el animal y la del ser humano, han facilitado también hacer una distinción entre miedo y ansiedad, lo cual es clave para su comprensión de estas emociones y para encontrar tratamientos más específicos. Una de las grandes ventajas de los modelos animales es que permiten tener un mayor control de diversas variables que puedan afectar la respuesta del sujeto, bajo estas condiciones, ha sido más sencillo diferenciar entre el miedo y la ansiedad. Se ha observado que, aún cuando los circuitos neurales de ambos son básicamente los mismos, la diferencia se encuentra en la intensidad de la respuesta, es decir, que lo que difiere entre ellas es la intensidad de activación más que su involucramiento (Hagenaars, 2014).

Es claro que el miedo y la ansiedad pueden presentase en diferentes momentos de una misma situación o como producto uno del otro, sin embargo, todos tienen una funcionalidad adaptativa para los individuos, siempre y cuando se mantengan dentro de los niveles fisiológicos normales (De Kloet Joëls, \& Holsboer, 2005; Pego et al., 2008). Cuando estos niveles se sobrepasan, ya sea de manera forzada o fortuita, es cuando se generan consecuencias importantes, a nivel conductual y cerebral (Ohman \& Mineka, 2001; Mineka, S. \& Ohman, 2002).

A la hora de utilizar un modelo animal, es importante tomar en cuenta las limitaciones que pueda tener con el fin de no arrojar conclusiones arriesgadas. Los modelos ayudan a replicar ciertas características, algunas conductuales, cerebrales, fisiológicas, a un nivel menos complejo en relación con el ser humano; lo que favorece una mejor comprensión de ciertos procesos debido al control de variables que caracteriza a los modelos animales y que es imposible en seres humanos debido a cuestiones éticas, costos, entre otros.

Para esto se deben considerar las variables que influyen en la reactividad emocional del animal, que van desde susceptibilidades propias de la especie o cepa, o de factores ambientales como eventos estresantes tanto pre- como post-natales, hasta el tipo de alojamiento, experiencias previas como el cuido materno y la edad, entre otras (Hau, 2003).

Tomando en cuenta la información presentada, se debe considerar el efecto del miedo y la ansiedad no sólo a nivel cognitivo, sino también sus repercusiones a nivel biológico. Es decir, que estas emociones pueden mejorar o empeorar condiciones físicas, por ejemplo alterando el funcionamiento normal del cuerpo y de regiones cerebrales involucradas; lo que es fundamental no sólo para modelos animales de miedo y ansiedad específicamente, sino que debe ser tomado en cuenta para cualquier modelo, ya que estas emociones son importantes moduladores de otros procesos cognitivos.

\section{Referencias}

Akirav, I. \& Maroun, M. (2007). The role of the medial prefrontal cortex-amygdala circuit in stress effects on the extinction of fear. Neural Plasticity, 23 (30), 1-11.

Archer (1973). Tests for emotionality in rats and mice: a review. Animal Behavior, 21, 205-235.

Arnsten, A. (2009). Stress signalling pathways that impair prefrontal cortex structure and function. Neurosci., 10, 410-422.

Becerra-García, A.M., Madalena, A.C., Estanislau, C., Rodríguez-Rico, J.L. \& Dias, H. (2007). Ansiedad y miedo: su valor adaptativo y maladaptaciones. Rev. Lationam. Psicol., 39, 75-81.

Bechara, A., Tranel, D., Damasio, H., Adolphs, R., Rockland, C., \& Damasio, A. R. (1995). Double dissociation of conditioning and declarative knowledge relative to the amygdala and hippocampus in humans. Science, 269, 1115-1118.

Bessa, J.M., Oliveira, M., Cerqueira, J.J., Almeida, O.F.X. \& Sousa, M. (2005). Age-related qualitative shift in emotional behaviour: Paradoxical findings after re-exposure of rats in the elvated-plus maze. Behav. Brain Res., 162, 135-142.

Blanchard, R., Blanchard, C., Griebel, G. \& Nutt, D. (2008). Introduction to the handbook on fear and 
anxiety. En: Blanchard, R., Blanchard, C., Griebel, G., Nutt,D. (Ed.) Handbook of anxiety and fear (pp. 3-7). Países Bajos: Elsevier.

Boschen, S. L., Wietzikoski, E. C., Winn, P., \& Cunha, C. D. (2011). The role of nucleus accumbens and dorsolateral striatal D2 receptors in active avoidance conditioning. Neurobiology of learning and memory, 96(2), 254-262.

Brenes, JC., Rodríguez, O. \& Fornaguera, J. (2006). Factor analysis of Forced Swimming test, Sucrose Preference test and Open Field test on enriched, social and isolated reared rats. Behavioural Brain Research, 169, 57-65

Bruchey, A.; Jones, C. \& Monfils, M. (2010). Fear conditioning by-proxy: Social transmission of fear during memory retrieval. Behavioural Brain Research, $214,80-84$.

Burman, M., Hamilton, K. \& Gewirtz, J. (2010). Role of corticosterone in trace and delay conditioned fear-potentiated startle in rats. Behavioral Neuroscience, 124, 294-299.

Campos, A.C., Fogaça, M.V., Aguiar, D.C. \& Guimaraes, F.S. (2013). Animal models of anxiety disorders and stress. Revista Brasileira de Psiquiatria, 35, 101-111.

Canteras, N.S. \& Graeff, F.G. (2014). Executive and modulatory neural circutis of defensive reactions: implications for panic disorder. Neuroscience Behavioral Reviews, 7634 (14), in press.

Castro, V. \& Landeira, J. (2008). Amygdaloid lesions produced similar contextual fear conditioning disruption in the Carioca high -and low-conditioned freezing rats. Brain Research, 1233, 137-145.

Carobrez A. \& Bertoglio, L. (2005). Ethological and temporal analyses of anxiety-like behavior: the elevated plus maze model 20 years on. Neuroscience and Behavioral Reviews, 29, 1193-1205

Choi, J.S., Cain, C.K \& LeDoux, J.E. (2010). The role of amygdale nuclei in the expression of auditory signaled two-way active avoidance in rats. Learn. Mem. 17, 139-147.
Davis, M., Falls, W., \& Gewirtz, J. (2000). Neural systems involved in fear inhibition: extinction and conditioned inhibition. In M. Myslobodsky \& I. Weiner (Eds.). Contemporary issues in modeling psychopathology, (pp. 113142). Estados Unidos: Elsevier.

De Kloet, E.R., Joëls, M \& Holsboer, F. (2002). Stress and the brain: from adatpation to disease. Neurosci., 6, 463-475.

Duval, F., González, F. \& Rabia, H. (2010) Neurobiología del estrés. Rev. Chil. Neuro-Psiquiat., 48, 307-318.

Dubovicky, M. (2004). Effect of chronic emotional stress on habituation processes in open field in adult rats. New York Academy of Sciences, 1018, 199-206.

Engel, K., Bandelow, B., Gruber, O. \& Wedekind, D. (2009). Neuroimaging in anxiety disorders. J. Neural Transm., 116, 703-716.

Everitt, B.J., Cardinal, R.N., Parkinson, J.A. \& Robbins, T.W. (2006). Appetitive behavior. Annals of the New York Academy of Sciences, 985, 233-250.

Fanselow, M.S. \& Ponnusamy, R. (2008). The use of conditioning tasks ro model fear and anxiety. En Blanchard, R.J., Banchard, D. C., Griebel, G \& Nutt, D.J. (eds.), Handbook of Anxiety and Fear (pp. 29-48). Países Bajos: Elsevier.

Fendt, M. \& Fanselow, M.S. (1999). The neuroanatomical and neurochemical basis of conditioned fear. Neurosci. Biobehav. Rev., 23, 743-760.

Fullford, A.J. \& Harbuz, M.S. (2005). An introduction to the HPA axis. En: Steckler, T., Kalin, N., Reul, J. (Ed.) Handbook of stress and the brain (3-23). Países Bajos: Elsevier

Gale, G., Anagnostaras, S, Godsil, B., Mitchell, S., Nozawa, T., Sage, J., Wiltgen, B. \& Fanselow, M. (2004). Role of the basolateral amygdala in the storage of fear memories across the adult lifetime of rats. The Journal of Neuroscience, 24, 3810-3815

Ganella, D.E \& Kim, J.H. (2014). Developmental rodent models of fear and anxiety: From neurobiology to pharmacology. British Journal of Pharmacology, 1, 5674. doi: 10.1111/bph.12643 
Gómez, C., Saldívar, A. \& Rodríguez, R. (2002). Modelos animales para el estudio de la ansiedad: una aproximación crítica. Salud Mental, 25, 14-24.

Green, M.R. \& McCormick, C.M. (2013). Effects of stressors in adolescence on learning and memory in rodent models. Hormones and behavior, 64, 364-379.

Grillon, C., Duncko, R., Covigton, M.F., Kopperman, L. \& Kling, M.A. (2007). Acute stress potentiates anxiety in humans. Biol. Psychiatry, 62, 1183-1186.

Gould (2009). The open field test. Mood and Anxiety Related Phenotypes in Mice. Neuromethods, 42, 1-20.

Hagenaars, M. (2014). Updating feeze: aligning animal and human research. Neuroscience and behavioral reviews, 47, 165-176.

Hau, J. (2003). Animal models. En Hau, J. \& VanHoosier G.L (eds.), Handbook of Laboratory Animal Science (pp. 8-16). EEUU: CRC Press.

Hogg, S. (1996). A review of the validity and variability of the elevated plus-maze as an animal model of anxiety. Pharmacology, Biochemistry and Behavior, 54, 21-30.

Kim, J., \& Whan, M. (2006). Neural circuits and mechanisms involved in Pavlovian fear conditioning: A critical review. Neuroscience and Biobehavioral Reviews, 30, 188-202.

Kloet, R., Jones, M., \& Holsboer, F. (2005). Stress and the brain: from adaptation to disease. Nature Reviews, 6, 63-75.

LaBar, K. S., LeDoux, J. E., Spencer, D. D., \& Phelps, E. A. (1995). Impaired fear conditioning following unilateral temporal labectomy in humans. Journal of Neuroscience, 15, 6846-6855.

LeDoux, J. (2000). Emotion circuits in the brain. Annual Reviews, 23, 155-184.

Levine, S. (2005). Stress: an historical perspective. En: Steckler, T., Kalin, N., Reul, J. (Ed.) Handbook of stress and the brain (3-23). Paises Bajos: Elsevier.

Lissek, S. (2005). Classical fear conditioning in the anxiety disorders: a meta-analysis. Behavior Research ant therapy, 43, 1391-1424.
Litvin, Y., Pentkowski, N.S., Pobbe, R.L., Blanchard, D.C. \& Blanchard R.J. (2008). En Blanchard, R.J., Banchard, D. C., Griebel, G \& Nutt, D.J. (eds.), Handbook of Anxiety and Fear (pp. 81-101). Países Bajos: Elsevier.

Marek, R., Strobel, C. Bredy, T. \& Saj, P. (2013). The amygdale and medial prefrontal cortex: partners in the fear circuit. The Journal of Physiology, 591, 2381-2391

Maren, S. (2001). Neurobiology of Pavlovian Fear Conditioning. Annual Reviews, 24, 897-931.

Masís-Calvo, M. Sequeira-Cordero, A. Mora-Gallegos, A. \& Fornaguera-Trías, J. (2013). Behavioral and neurochemical characterization of maternal care effects on juvenile Sprague-Dawley rats. Physiology and Behavior, 118, 212-217.

McNaugthon, N. \& Zangrossi, H.,Jr. (2008) Theoretical approaches to the modeling of anxiety in animals. En Blanchard, R.J., Banchard, D. C., Griebel, G \& Nutt, D.J. (eds.), Handbook of Anxiety and Fear (pp. 11-28). Países Bajos: Elsevier.

Mineka, S. \& Ohman, A. (2002). Phobias and Preparedness: The Selective, Automatic, and Encapsulated Nature of Fear. Society of Biological Psychiatry, 52, 927-939.

Moscarello \& LeDoux (2013). Active avoidance learning requires prefrontal suppression of amygdala-mediated defense reactions. Journal of Neuroscience, 3815-3823.

Moustafa, A.A., Gilbertson, M.W., Orr, S.P., Herzahllah, M.M., Servatius, R.J. \& Myers, C.E. (2013). A model of amygdala-hippocampal-prefrontal interaction in fear conditioning and extinction in animals. Brain and Cognition, 81, 29-43.

Ohman, A. \& Mineka, S. (2001). Fears, phobias and preparedness: toward an evolved module of fear and fear learning. Psychological Review, 108, 483-522.

Ossenkopp \& Mazmanian (1985). The principle of aggregation in psychobiological correlational research: an example from the open field test. Animal learning and behavior, 13, 339-344 
Pang, K. C., Jiao, X., Sinha, S., Beck, K. D., \& Servatius, R. J. (2011). Damage of GABAergic neurons in the medial septum impairs spatial working memory and extinction of active avoidance: effects on proactive interference. Hippocampus, 21, 835-846.

Pego, J., Morgado, P., Pinto, L., Cerqueira, J., Almeida, O. \& Sousa, N. (2008). Dissociation of the morphological correlates of stress-induced anxiety and fear. European Journal of Neuroscience, 21, 1503-1516.

Pellow, S., Chopin, P., File, S. \& Briley, M. (1985). Validation of open closed arm entries in an elevated plus maze as a measure of anxiety in the rat. Journal of Neuroscience Methods, 14, 149-167.

Pleskacheva, M. (1996). Temporal characteristics of grooming in an open field in two strains of rats. International Journal of Comparative Psychology, 9, 105-116.

Prut, L\&Belzung, C. (2003). The open field as a paradigm to measure the effects of drugs on anxiety-like behaviors: a review. European Journal of Pharmacology, 463, 3-33.

Radulovic, J., \& Tronson, N. C. (2010). Molecular specificity of multiple hippocampal processes governing fear extinction. Rev. Neurosci., 21, 1-18.

Rodgers, R. \& Dalvi, A.(1997) Anxiety, Defense and the elevated plus-maze. Neuroscience and Behavioral Reviews, 6, 801-810.

Rodrigues, S.M., LeDoux, J.E. \& Sapolsky, R.M. (2009). The influence of stress hormones on fear circuitry. Annals Review of Neuroscience, 32, 289-313.

Rohra, D.K. \& Qazi, Y. (2008). Reliability of rodent models. En Conn, P.M. (ed.), Sourcebook of Models for Biomedical Research (pp. 213-220). EEUU: Humana Press.

Roth, K. A. (1979). Stress, behavioral arousal and open field activity-a reexamination of emotionality in the rat. Neuroscience and biobehavioral reviews, 3, 247-263

Roozendaal, B., McEwen, B., \& Chattarji, S. (2009). Stress, memory and the amygdale. Nature Reviens, 6, 423-433.
Sandi, G. \& Pinelo-Nava, M.T. (2007). Stress and memory: behavioral effects and neurobiological mechanisms. Neural Plast., 78970, 1-20.

Seligman, M. E. (1971). Phobias and preparedness. Behavior Therapy, 2, 307-320.

Sequeira-Cordero, A., Masís-Calvo, M., Mora-Gallegos, A. \& Fornaguera-Trías, J. (2013). Maternal behavior as an early modulator of neurobehavioral offspring responses by Sprague-Dawley rats. Behavioural Brain Research, 237, 63-70.

Shanks, N., Greek, R. \& Greek, J. (2009). Are animal models predictive for humans? Philos., Ethics, and Humanit. Med., 4, 1-20.

Sierra, JC., Ortega, V., \& Zubeidat, I. (2003). Ansiedad, angustia y estrés: tres conceptos a diferenciar. Revista Mal-estar y subjetividade, 3, 10-59.

Suárez, S.D. (1981). An ethological analysis of open field behavior in rats and mice. Learning and motivation, 12, 342-363.

Sullivan, G.; \& LeDoux, J. (2004). Synaptic Self. En: Gorman, J. (Ed). Fear and anxiety (1-22). American Psychopathological Association, (pp. 1-22). New York

Walf, A.A. \& Frye, C.A. (2007). The use of the elevated plus maze as an assay of anxiety-related behavior in rodents. Nature Protocols, 2 , 322-328.

Walsh, R.A., \& Cummins, R. N. (1976). The open field test: a critical review. Psychological bulletin, 83, 482-504

Wendler, E. (2014). The roles of the nucleus accumbens core, dorsomedial striatum, and dorsolateral striatum in learning: Performance and extinction of Pavlovian fear-conditioned responses and instrumental avoidance responses. Neurobiology of learning and memory, 109, 27-36

Zeidner, M. \& Matthews, G. (2011). Anxiety. New York: Springer. 